\title{
Premios Congreso SEDEN Bilbao 2013
}

Premio Izasa Hospital. "Accesos Vasculares y Nuevas Tecnologías"

Estudio comparativo de dos tipos de agujas en hemodiafiltración de alta eficacia

Ana Vanessa Fernández Martínez, Verena Romero Willhoft, Laura Pérez Valencia, Alicia Moreno Vallejo, Tania Ortega Gómez, Salvadora Soto Ureña.

Fresenius Medical Care Services Cartagena y San Pedro del Pinatar. Cartagena. Murcia

Premio B. Braun Medical. "Personalización del Tratamiento de Hemodiálisis"

Resultados de un programa de ejercicio físico combinado con electroestimulación neuromuscular en pacientes en hemodiálisis

Gorka Iza Pinedo, Anna Junqué Jiménez, Esther Tomás Bernabeú, Inés Luceño Soler, Oscar Paz López, Marisa Lavado Sempere.

Servicio de Nefrología Hospital de Terrassa. Consorci Sanitari Terrassa. Barcelona

Premio Fresenius Medical Care para Enfermería. "Hemodiafiltración en Línea"

Estudio comparativo en pacientes en hemodiafiltración on-line postdilucional de alta eficacia con diferentes flujos de líquido dializante con tiempo programado y tiempo real

Sonia Aznar Barbero, Ramón Bel Cegarra, Ma ría Magdalena Badallo Mira, David Pagán Escribano, Asensio Bartolomé García Nicolás.

Hospital de Santa Lucía de Cartagena. Murcia
Premio Fresenius Medical Care para Enfermería."Diálisis Peritoneal"

Evaluación de los conocimientos prácticos de los pacientes prevalentes en diálisis peritoneal

Beatriz Peláez Requejo, Mónica Fernández Pérez, Miguel Núñez Moral, Isabel González Díaz, Alejandra Méndez González, Aurora Quintana Fernández Hospital Universitario Central de Asturias (HUCA). Oviedo

Premio Bellco "Al Mejor Trabajo de Investigación sobre Terapias Convectivas"

Análisis comparativo entre hemodiafiltración en línea frente a hemodiafiltración con reinfusión endógena: parámetros clínico-técnicos e inflamatorios Carmen Ramírez Moreno, Irene Torollo Luna, $\mathrm{M}^{\mathrm{a}}$ Dolores López Zamorano, Concepción Guisado Segador, Francisco Salas Cardador, Mateo Alcántara Crespo Centro Periférico Perpetuo Socorro. Córdoba 